mental cross sections, but not very well with the details of the angular distributions, as shown in the figure. This discrepancy may indicate the importance of higher order effects, in particular the two-step reactions that proceed through an intermediate state.

The detection of this reaction opens up a new area of nuclear reaction and nuclear structure studies, and in particular suggests that it may be useful to look for the $\left({ }^{3} \mathrm{He}, \alpha^{*}\right)$ and (d, $\left.\alpha^{*}\right)$ reactions as well.

\section{Tertiary plate motions}

\section{from Peter J. Smith}

THE relative angular velocities of the Earth's lithospheric plates are now quite well known. The 'absolute' angular velocities of the plates (that is their velocities relative to the mesosphere) are known much less precisely; but if one accepts, with Solomon et al. (Geophys. J. 42, 766; 1975), that absolute linear velocities can now be specified to within $1-2 \mathrm{~cm} \mathrm{yr}^{-1}$, then it is possible to make three statements, or groups of statements, about their relationships. First, there is a good, but not perfect, general correlation between the velocity of a plate and the fraction of the plate occupied by continent; the greater the proportion of continent the lower is the velocity. At the extremes, predominantly oceanic plates have velocities in the range $7-10 \mathrm{~cm} \mathrm{yr}^{-1}$ whereas predominantly continental plates generally move at $0-2 \mathrm{~cm} \mathrm{yr}^{-1}$. Second, plates being subducted along a major proportion of their boundaries all have high velocities. And third, lithosphere in the equatorial half of the Earth has a higher r.m.s. velocity that that in the two polar quarters.

These three statements give rise, in turn, to three questions. Do continental plates move more slowly because there is a greater viscous drag at the base of the continental lithosphere than beneath the oceanic lithosphere? Do subducting plates have high velocities because of a major force exerted by the downgoing lithospheric slabs? And does the polar-equatorial velocity differential arise because of a connection between plate motions and the Earth's rotation? It is tempting to allow circumstantial evidence to answer all three questions in the affirmative, or at the very least to argue that the relationships indicated by the three statements have underlying physical causes. But equally, such relationships could well be coincidental. How, then, can the issue be decided?

The ideal way, as Solomon et al. (J. geophys. Res. 82, 203; 1977) point out, would be "to observe a number of global plate systems, each with very different plate geometries but obeying similar physical laws". But that is obviously not possible; so Solomon and his colleagues have done the next best thing in studying the behaviour of the Earth's plates as they were arranged $55 \mathrm{Myr}$ ago. Using the plate boundary map for that period already constructed by Jurdy and Van der Voo (J. geophys. Res. 79, 2945; 1974) together with published values for finite plate rotations, they first determined the relative plate velocities. Absolute velocities were then calculated assuming no net torque to be exerted on the lithosphere for a series of models having driving forces symmetrical about the plate boundaries and a plateretarding viscous drag at the base of the lithosphere.

Needless to say, such determinations are more complicated that they are made to sound here and involve numerous assumptions, approximations and simplifications that may later give critics cause to make trouble. Taken at face value, however, the results suggest that it may be dangerous to invoke particular physical processes purely on the basis of the behaviour of the plates as they are arranged now. For one thing, with the plates as they were $55 \mathrm{Myr}$ ago there is apparently no correlation between r.m.s. absolute linear velocity and the fraction of the plate comprising continent, assuming the viscous drag to be uniform at the base of both the continental and oceanic lithospheres. Nor is there a clear division between fast-moving predominantly oceanic plates and slowmoving predominantly continental plates. For example, $55 \mathrm{Myr}$ ago the Eurasian plate, $93 \%$ of which is continent, had a velocity about equal to that of the Pacific plate. It is true that the Eurasian plate is much slowed down if the uniform drag model is replaced by one in which there is drag only beneath the continental lithosphere; but not only would the latter model violate palaeomagnetic and other constraints, it would actually produce an increase in most continental velocities.

Fifty-five million years ago, plates with major subduction zones were not all moving rapidly either. The Farallon plate, for example, with a large fraction of its boundary under active subduction, was moving at less than half of the rate of the fastest (Kula) plate. On the other hand, the conclusion to be drawn from the polar-equatorial velocity differential is more ambiguous.
The differential undoubtedly existed, albeit in a much weaker form than at present. But whereas the coordinate pole giving the maximum equatorial r.m.s. velocity is now almost coincident with the geographic pole (apparently strengthening the supposition that plate motions and the Earth's rotation are linked), it is $60^{\circ}$ from the geographic pole for the older plate distribution. Solomon and his colleagues therefore conclude that the case for a relationship between the Earth's rotation and plate velocities is "not particularly compelling" at $55 \mathrm{Myr}$ and thus, by implication, at present.

On balance, then, this new evidence suggests that the three original questions should be answered negatively; and the fact that affirmative answers are consistent with current plate motions would appear to be a coincidence. But that is not all, for Solomon et al. have also been able to bring their analysis to bear on the controversy over whether or not the supposed mantle plumes from which hot spots are derived are fixed in the mantle. By comparing their newly-determined absolute plate motions with observed hot spot traces, they find the fits between azimuths to be variable. For hot spot traces within the Indian and African plates the fits are within expected errors, but there are serious misfits for Pacific plate hot spots and the Icelandic hot spot. In short, not all mantle plumes appear to be fixed with respect to each other and thus not all can be fixed with respect to the mantle.

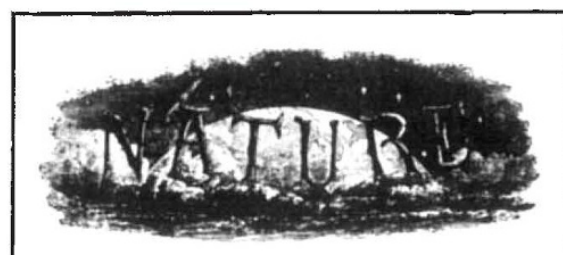

\section{A hundred years ago}

Hrs Majesty, the Emperor of Brazil, observed the eclipse of the moon on the evening of the 27th, at the Arcetri Observatory. The Emperor took a very lively interest in the phenomenon and discussed with acuteness the hypothesis with which Prof. Tempel, the astronomer, and Prof. Echert tried to explain the varying shades and colours in which the moon appeared during the different phases of obscuration. On Monday last his Majesty assisted at a meeting of the Anthropological Society, when Prof. Mantegazza made some interesting remarks on several Maori skulls, and Prof. Giglioli read an elaborate paper on the ethnology of Brazil.

From Nature 16, March 8, 416; 1877. 